\title{
Prompt Gamma Activation Analysis of Impurity and Elemental Stability for Two SU-8 Organic Polymer Samples Having Different Solidification Pretreatment
}

\author{
M.E. Heaton ${ }^{1}$, M. Rogante ${ }^{*, 2}$, Zs. Kasztovszky ${ }^{3}$ and D. Denieffe ${ }^{4}$ \\ ${ }^{I}$ Optical and Semiconductor Devices Group, Dept of Electrical and Electronic Engineering, Imperial College London, \\ South Kensington, London SW7 2AZ \\ ${ }^{2}$ Rogante Engineering Office, Contrada San Michele, 61, P.O. Box 189, 62012 Civitanova Marche, Italy \\ ${ }^{3}$ Institute of Isotopes, Hungarian Academy of Sciences, H-1121 Budapest, Konkoly Thege 29-33 \\ ${ }^{4}$ School of Engineering, Institute of Technology Carlow, Kilkenny Rd, Republic of Ireland
}

\begin{abstract}
In this paper, two samples of SU-8, a photo polymer consisting of Gamma-Butyrolactone (GLB) mixed with both Triarylsulfonia and Hexafluroantimonate considered for a Prompt Gamma Activation Analysis (PGAA) diagnostic study. The SU-8 was formed into two organic polymer microturbines which were then given different solidification pretreatment using varying durations of heat baking and Ultra-Violet (UV) crosslinking. In addition to determining the major components of the analyzed samples, some trace elements were also identified, and all the compositions were compared. The measurements were performed at the Budapest Research Reactor (BRR). The obtained results have advanced the industrial applicability of the adopted technique in the considered sector of polymer analysis, because the PGAA has been applied in the case of the polymer of the microturbines. The results obtained are complementary to data achieved by other techniques.
\end{abstract}

Keywords: Microturbine, Micro airflow-blades, Polymerisation, UV Crosslinking, PGAA, Industrial Application of Neutron Techniques.

\section{INTRODUCTION AND TARGET AIMS OF USING PGAA FOR SU-8 POLYMER ANALYSIS}

The microturbine is a new device to pioneer either gas or airflow measurement using the low pressure-head characteristics of axial-flow on the micro-scale. The microturbine can respond to ambient airflow because it flows through the device axially via its large exposed top surface area. The microturbine device is being developed from both a scientific and an industrial point of view [1-3]. The microturbine samples considered for the PGAA (i.e., Prompt Gamma Activation Analysis) investigation were produced using excimer laser fabrication with mask-dragging to profile the optimally smooth airflow contours of the blades.

The maximum output of the microturbine is $\sim 160 \mathrm{mV}$ at $132 \mathrm{krpm}$. This output requires a Nitrogen flow rate of 80 litres per minute, which corresponds to a mass flow rate of $1.5 \mathrm{~g} / \mathrm{s}$, and an angular velocity of $21.53 \mathrm{~m} / \mathrm{s}$. If the microturbine is run at this maximum it causes a lot of stress on each of the 31 blades of the $13 \mathrm{~mm}$ diameter microturbine dics. The blades are $1.5 \mathrm{~mm}$ in length by $1 \mathrm{~mm}$ in height and $0.05 \mathrm{~mm}$ in thickness, as seen in Fig. (1).

The microturbines tested in this experiment were part of a microelectrical generator that could provide an airspeed

*Address correspondence to this author at the Rogante Engineering Office, Contrada San Michele, 61, P.O. Box 189, 62012 Civitanova Marche, Italy; Tel: +39 0733 775248; Fax: +39 0733 775248;

E-mail: main@ roganteengineering.it reading and enough net power to transmit data. This turbine produced enough extra power via the generator to power its electronics to device. This self powering aspect meant the device could be used in long term applications requiring wireless operation and hence no need for a battery change. The main applications of the device are in remote environmental monitoring systems, wireless flowmetry sensors and airflow metrology e.g. aeronautics. The microturbine could also be used for micro-cooling in electronic systems (aerospace applications) if operated as a micro-motor.

The power output requirements of the microturbine meant that the material from which it was made, commercially known as SU-8, has to be very consistent and strong on the micro-scale of the blades. PGAA was considered as a means to test the SU-8 for any changes in its elemental composition, as such could compromise its strength.

The SU-8 constitutive material for the turbine preforms and hence the finished microturbines is an organic resin solution that forms a negative photo resist containing an epoxy resin organic solvent and gamma-Butyrolactone. The percentages of the constituents of the resin are 35-75 percent Epoxy Resin (CAS: 28906-96-9) and 22-60 percent Gamma Butyrolactone (CAS: 96-48-0) mixed with 1-5 percent Triarylsulfonia/Hexafluroantimonate Salt (CAS: 89452-37-9)CAS: 71449-78-0) and Propylene Carbonate (CAS: 108-327) [3].

This substrate material which is a viscous clear liquid in its natural manufactured state was initially exposed to UV to 
prepare it for oven drying and final hardening (curing or polymerisation) in order to make the preforms for the microturbines as seen in Fig. (1a). The material has to be solidified using UV radiation and heat to cure and crosslink it. Cured SU-8 polymer is very light and strong, but brittle in the hardened state.

In the semitransparent hardened state, as in the case of the finished microturbine shown on Fig. (1b) the SU-8 consists of a C-C-O (carbon-carbon-oxygen matrix) in the form of repeating triangles. The $\mathrm{O}$ occasionally forms $\mathrm{OH}$ molecules when heated, which join with other hydroxyl molecules to form water [3, 4].

This material has a structure which can interact with neutrons due to its ability to crosslink once in the hardened/dried state. This neutron compliance is a positive aspect of the SU8, unlike for example PMMA polymer material, which in not forming crosslinks, stays in long chain molecules and would be broken into monomers under neutron exposure.

The micro blades undergo very high drag on their surfaces when the microturbine is run at its optimum power output. This is due to the effect of scaling the turbine's dimensions down to the micro-scale. Size reduction causes disproportionate volume to surface area scaling effects (area is more affected as the volume reduces). Thus the blades have to be very thin, or ideally of zero thickness to minimize such drag. They were produced from SU-8 polymer as it has the property of being a very strong photoresist suited to microscale fabrication.

The turbines were made to be the first having axial airflow on the microscale, for response to low air pressure. The blades have to be strong along their radial lengths so as not to fracture under air pressure passing through and on top of them. Impurities in the resin matrix may influence the material quality and hence the strength of the finished microturbines as seen in Figs. (1b) to (1d). Fig. (1a) is a preform turbine made of hardened and crosslinked SU-8. The final turbine seen in Fig. (1b) is made by laser machining the preform in Fig. (1a).

Figs. (1c) and (1d) show processing damage in the form of trenches cut into the corners of the upper and lower surfaces of the turbine blades. This is due to increased heat conduction during laser machining in the blade corners, and may cause defects or flaws in the subsurface of the SU-8. It is best to have the SU- 8 in the most consistent composition state possible at the stage of making the preform turbine.

PGAA was used to measure any changes in the percentages of all the elements that made the SU-8, as may be caused by the heat and UV curing radiation. Also, increased heat conduction into the corners of the blades may change the $\mathrm{H}$ or $\mathrm{O}$ elements and hence affect the strength of the blades. The effect of increased corner heat can be seen in Fig. (1c) where the blade ends have become undercut. Additionally, the heat pretreatment needed to dry, harden and cure the SU-8 may cause some element differences resulting in one turbine being different to another turbine. Two turbines were used to make a complete micro airflow sensor, in that one turbine acted as a stator to direct potentially very high velocity air or gas flow onto the rotor turbine. It was also of interest to examine how effective PGAA was for investigating a polymer material like SU-8.

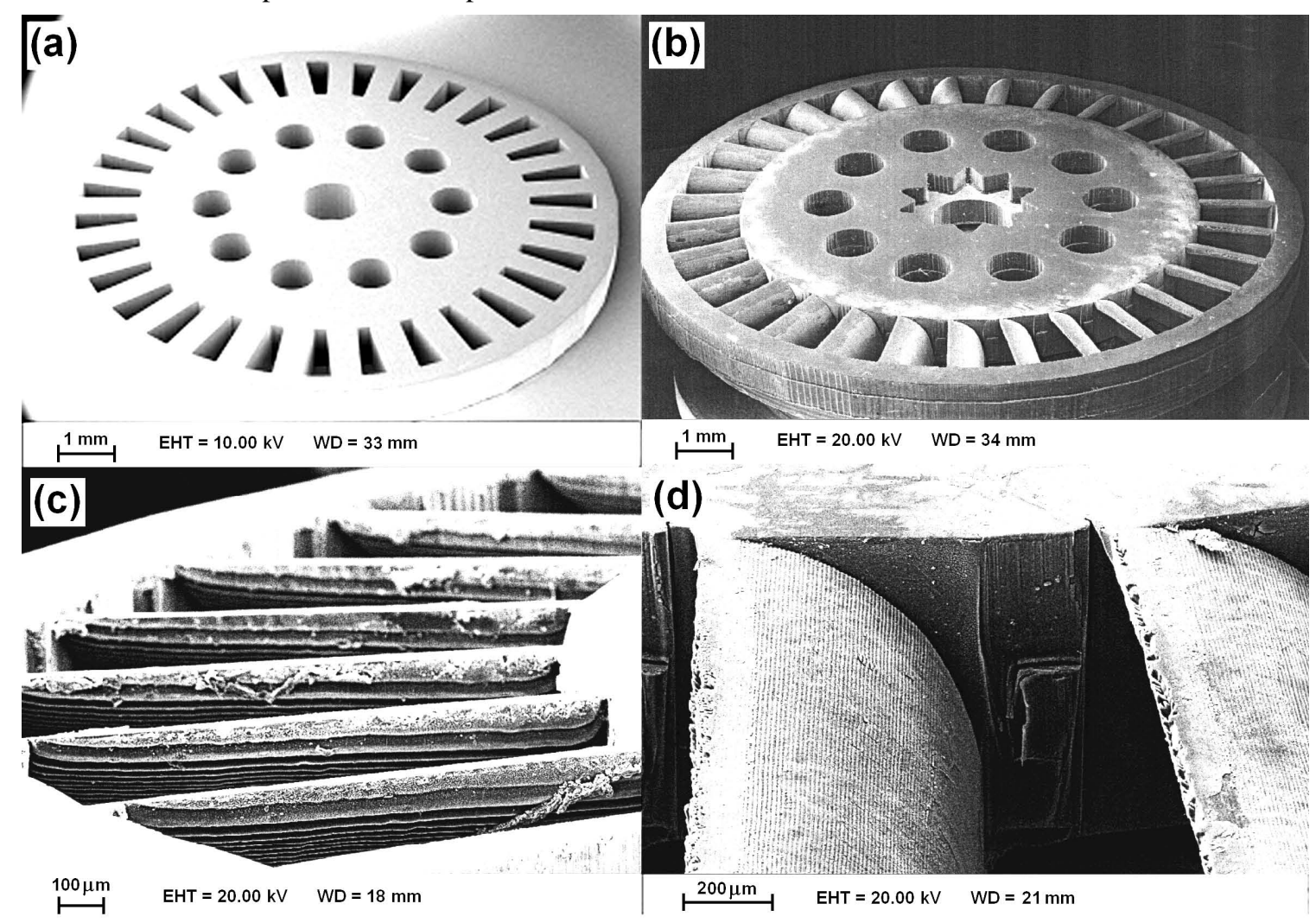

Fig. (1). The investigated SU-8 organic polymer microturbine samples where (a) is a turbine preform, (b) a finished turbine, (c) a series of turbine edges, and (d) the smooth surface detail of two turbine blades finished using laser machining. 


\section{PROCEDURE TO OPTIMISE THE PGAA IN- STRUMENTATION SETUP}

Neutron investigations have recently become an increasingly significant probe for materials across a wide range of disciplines, and can reveal significant properties about materials. Neutrons are becoming ever more useful in the nondestructive characterisation of materials and components of industrial interest. The industrial applications of neutron techniques are also being developed in many new industrial sectors [4-7].

PGAA is a relatively new non-destructive nuclear analytical method based on the detection of characteristic prompt gamma photons that originate in $(n, \gamma)$ nuclear reactions. The principles of the method have been well known for decades, and industrial applications are currently in development. However, routine application of the method is not so frequent because of the relatively low number of available laboratories equipped with guided neutron beams. Every atomic nuclei, apart from ${ }^{4} \mathrm{He}$, may undergo a $(\mathrm{n}, \gamma)$ reaction with different probabilities. The energies of the emitted gamma photons are characteristic for each given isotope, while the intensities of the gamma peaks are proportional to the amount of a given isotope. This phenomenon allows the use of a quantitative elemental (isotopic) analysis method known as PGAA or PGNAA [9]. PGAA gives information on the sample as a whole. Neutrons can penetrate the surface and lower layers of the material, so PGAA cannot distinguish between the "bulk" and "surface" composition of a sample.

The PGAA experiment on these SU-8 polymer samples is a first attempt to apply PGAA to detect trace elements in an organic matrix, and one of the first such industrial applications of PGAA [8]. The measurements were performed at the PGAA station using the 10 MW Budapest Research Reactor (BRR), as seen in Fig. (2).

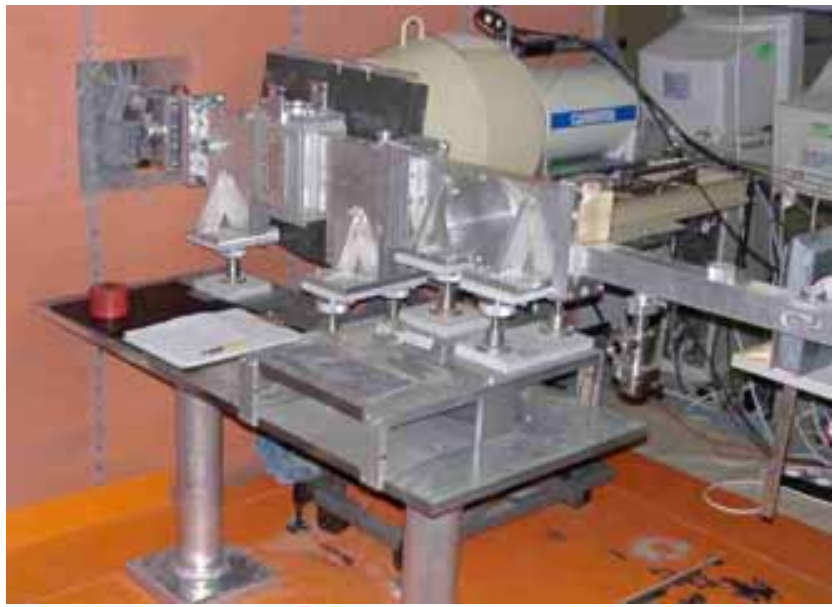

Fig. (2). The Prompt Gamma Activation Analysis Instrument at the BRR.

The PGAA instrument was placed at the end of a 30 metre long horizontal guide. The neutrons, which exit the reactor core, are moderated by a liquid $\mathrm{H}$ cell, and are cooled down to $20 \mathrm{~K}$. The " $1 / v$ " dependence of the neutron absorption cross-section makes the sensitivity of the method in- crease by a factor of twenty, compared to the thermal sensitivity of the beam. Until mid 2006, the thermal equivalent flux of the cold beam was only $5 \cdot 10^{7} \mathrm{~cm}^{-2} \mathrm{~s}^{-1}$, but following the first two upgrades of the neutron guides, between January and October 2007, a thermal equivalent flux of $7 \cdot 10^{7} \mathrm{~cm}^{-2} \mathrm{~s}^{-1}$ was achieved. A final third upgrade later allowed the use of an intensity of $1.2 \cdot 10^{8} \mathrm{~cm}^{-2} \mathrm{~s}^{-1}$.

\section{EXPERIMENTAL PROCEDURE, RESULTS AND DISCUSSION}

The investigated samples were mounted on an aluminium frame with Teflon strings, as seen in Fig. (3). The sample holder chamber was evacuated in order to minimize the spectral background.

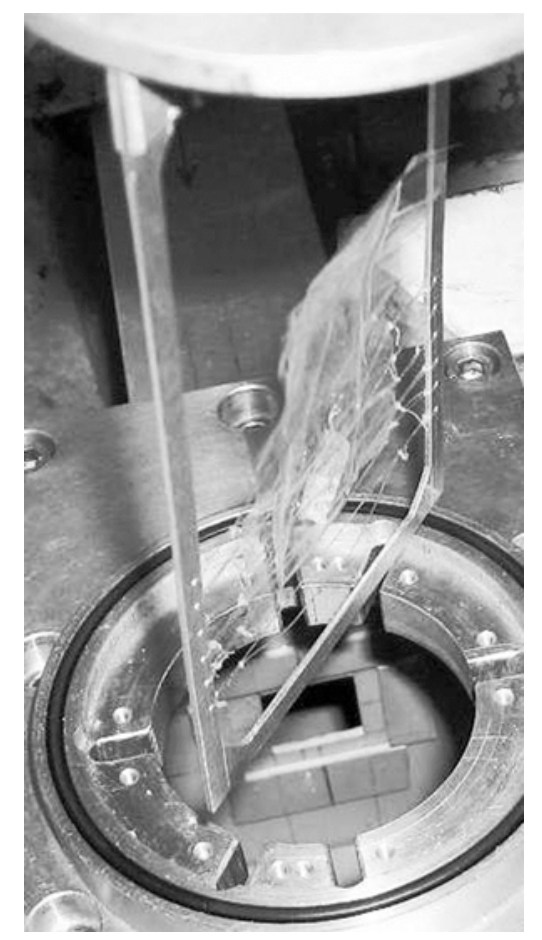

Fig. (3). SU-8 microturbine sample fastened with Teflon strings onto an aluminium frame for introduction into the PGAA measurement chamber.

A $2 \times 2 \mathrm{~cm}$ cold beam was applied for irradiation. The irradiation time was chosen to achieve the counting statistics of necessary confidence limits. In practice sample one was irradiated for about $14000 \mathrm{~s}$, while sample two was irradiated for almost $20000 \mathrm{~s}$. The background effects originated from $(\mathrm{n}, \gamma)$ reactions within the constituent materials (i.e., $\mathrm{H}, \mathrm{C}, \mathrm{Cl}$, $\mathrm{Al}, \mathrm{Fe}$ and $\mathrm{Pb}$ ) were calculated, as listed later in Tables $\mathbf{1}$ and 2.

Parallel to the irradiation, the prompt gamma spectra were recorded with a specially designed detector system. This consisted of a 27 percent High Purity Germanium detector surrounded with Bismuth Germanate scintillators which are dedicated to performing the Compton-suppressed measurement mode. The signals coming from the detectors are processed using a $\mathbf{S} 100$ multichannel analyser. The spectra were evaluated using a Hypermet-PC having software developed at the Institute of Isotopes of the BRR; the element identification is based on a prompt-gamma library [10]. 
The quantitative analysis is based on the following considerations. The detected gamma ray intensity $A_{E}$ is directly proportional to the mass $m$ of a given element, the analytical sensitivity $S$ and the measurement time $t$, such that $A_{E}=m \cdot S \cdot t$. The detected count rate $\left(\frac{d N_{P}}{d t}\right)$ in a given gamma peak is proportional to the number of nuclei emitting the gamma photons of a given energy. It can be calculated, as in the following equation:

$$
\frac{d N_{P}}{d t}=\int_{V} \int_{E_{n}=0}^{\infty} \frac{\mu(\mathbf{r}) N_{A v}}{M} \sigma_{\gamma}\left(E_{n}\right) \Phi^{\prime}\left(E_{n}, \mathbf{r}\right) \varepsilon^{\prime}\left(E_{\gamma}, \mathbf{r}\right) d E_{n} d \mathbf{r}
$$

where $N_{P}$ is the peak area, $\mu(\boldsymbol{r})$ is the density of the element of interest in the point $\mathbf{r}, N_{A v}$ is the Avogadro number, $M$ is the atomic mass of the element, $\Phi^{\prime}\left(E_{n}, \mathbf{r}\right)$ is the local neutron flux and $\varepsilon^{\prime}\left(E_{\gamma}, \mathbf{r}\right)$ is the detector efficiency.

A few simplifications, in practice, can be introduced. For example, $\sigma_{\gamma}$ is the partial gamma ray production cross section, and can be defined as:

$\sigma_{y}=\sigma_{0} \cdot I_{y} \cdot \theta$

where $\sigma_{0}$ is the thermal neutron absorption cross section, $I_{\gamma}$ is the probability of gamma ray emission and $\theta$ is the isotopic abundance.

If it is supposed that the we have a small, thin and homogeneous sample, and that the detector efficiency is independent of the sample position (it is in a fixed position), then the thermal equivalent flux $\left(\phi_{0}\right)$ is defined so as to have the same reaction rate, as below:

$$
\begin{aligned}
R & \equiv N \int_{E_{n}=0}^{\infty} \Phi\left(E_{n}\right) \cdot \sigma\left(E_{n}\right) d E_{n}=N \int_{E_{n}=0}^{\infty} v \cdot n\left(E_{n}\right) \cdot \frac{\sigma_{0} v_{0}}{v} d E_{n}= \\
& =N \cdot \sigma_{0} \int_{E_{n}=0}^{\infty} v_{0} \cdot n\left(E_{n}\right) d E_{n}=N \cdot \sigma_{0} \cdot \Phi_{0}
\end{aligned}
$$
tion:

Such that the peak area may be calculated from the equa-

$$
N_{P}=\rho \cdot t_{m}=m \cdot S \cdot t_{m}=\frac{m N_{A v}}{M} \Phi_{0} \sigma_{\gamma} \varepsilon\left(E_{\gamma}\right) t_{m}
$$

And hence the sensitivity may be given by:

$S=\frac{N_{A}}{M} \cdot \theta \cdot \sigma_{0} \cdot I_{\gamma} \cdot \Phi_{0} \cdot \varepsilon\left(E_{\gamma}\right)$

The analytical sensitivity $S$ is expressed in units of counts $\cdot \mathrm{s}^{-1} \cdot \mathrm{g}^{-1}$, as seen in eq. (5), and is proportional to the neutron capture cross-section of the following nuclear constants, the nucleus $\sigma_{0}$, the isotopic abundance $\theta$, and the gamma yield $I_{r}$ as well as to the neutron flux $\Phi_{0}$ and the detector efficiency $\varepsilon\left(E_{\gamma}\right)$, which are characteristics of the measuring system. According to eq. (3), lower energy (cold) neutrons mean higher thermal equivalent flux, which according to eq. (5) means better sensitivities.

Other symbols in eq. (5) are Avogadro's number $N_{A}$, and the atomic mass $M$ of any given element. The mass ratios, or equivalently the weight-percentage ratios of arbitrary ele- ments " $X$ " and " $Y$ " are independent of both the actual amount of each sample and of the exact neutron flux, and can be calculated from peak area ratios and sensitivity ratios as follows:

$$
\frac{w_{X}(\%)}{w_{Y}(\%)}=\frac{m_{X}}{m_{Y}}=\frac{A_{X}}{A_{Y}} \cdot \frac{S_{\gamma, Y}}{S_{\gamma, X}}
$$

The sensitivities for the most intensive prompt-gamma lines of all the chemical elements were determined by internal standardisation measurements at the BRR and are collected in a new gamma-ray spectrum catalogue for PGAA [10].

In order to calculate the element concentrations, the 25 most intensive gamma lines of each element are used, assuming such lines and found. During the calculations, gamma lines, which suffer from peak interference (i.e. overlapping the nearby peaks) are eliminated from the determination of all the element concentration.

In Table 1 the most intensive gamma line data for the elements are reported. These resulted from line data that were identified in the samples. The symbols and atomic masses of the isotopes, the order of peak intensities, relative peak areas, the characteristic gamma energies and their uncertainties, the partial gamma ray production cross sections and the calculated sensitivities are listed. When all major elementary components are determined by PGAA, it is not necessary to measure a standard comparator material with the sample, since the concentrations can be determined using equations (6) and (7), with (7) being stated for completeness:

$\sum_{i} w_{i}(\%)=100$

This criterion is fulfilled in the case of the SU-8 samples one and two. PGAA is rather non sensitive for light elements like $\mathrm{C}$ and $\mathrm{O}$. In the case of these two samples, $\mathrm{C}$ and $\mathrm{O}$ make the major part of their elemental compositions. In addition to finding the major components of $\mathrm{H}, \mathrm{C}, \mathrm{O}$ and $\mathrm{F}$, the PGAA investigation also allowed us to quantify other traces, e.g. $\mathrm{S}, \mathrm{Cl}, \mathrm{Fe}, \mathrm{Sb}$ and $\mathrm{Pb}$, as shown in Table 2.

Table 2 shows the compositions of samples one and two in both atomic percentage and weight percentage. Other trace contaminants, if there were any, fell under the detection limits of PGAA. Comparing samples one and two using PGAA did not reveal any significant difference as can be seen in Fig. (4).

The achieved similar elemental composition for the SU-8 samples having different pretreatment is a good result, as a composition loss or difference could affect the mechanical properties of the polymer. It is often assumed that SU-8 absorbs and loses $\mathrm{H}$ and $\mathrm{O}$, especially if heated and effectively boiled away, particularly in the corners of the blades where excess heats builds up during heat curing and laser machining. The high percentages of the elements $\mathrm{H}$ and $\mathrm{O}$ are also assumed to rise and fall with exposure to humidity over a period of days.

The two samples were exposed to different heat and UV durations but show no discernible element percentage difference, including none even in their very high $\mathrm{H}$ and $\mathrm{O}$ 
Table 1. List of the Most Intensive Gamma Line Data for the Elements Identified in the Samples, Showing the Symbols and Atomic Masses of the Isotopes, the Order of Peak Intensities, the Relative Peak Areas, the Characteristic Gamma Energies and their Uncertainties, the Partial Gamma Ray Production Cross Sections and the Calculated Sensitivities

\begin{tabular}{|c|c|c|c|c|c|c|c|}
\hline El. & $\mathbf{A}$ & No. & Rel. Area & E (keV) & dE $(k e V)$ & $\sigma_{\gamma}$ & Sens. (cps/mg) \\
\hline $\mathrm{H}$ & 1 & 1 & 100 & 2223.3 & 0.019 & 0.333 & 3.4 \\
\hline $\mathrm{C}$ & 12 & 2 & 60.55 & 4945.302 & 0.067 & 0.0028 & 0.0011 \\
\hline $\mathrm{C}$ & 12 & 3 & 38.02 & 3684.016 & 0.069 & 0.00121 & 0.00066 \\
\hline $\mathrm{O}$ & 16 & 3 & 50.79 & 2184.381 & 0.039 & 0.00015 & 0.000096 \\
\hline $\mathrm{O}$ & 16 & 4 & 7.01 & 3272.109 & 0.069 & 0.000035 & 0.000015 \\
\hline $\mathrm{F}$ & 19 & 1 & 100 & 1633.602 & 0.015 & 0.0093 & 0.0064 \\
\hline $\mathrm{F}$ & 19 & 2 & 99.00 & 583.493 & 0.022 & 0.0034 & 0.005 \\
\hline $\mathrm{S}$ & 32 & 3 & 16.16 & 5420.241 & 0.1 & 0.32 & 0.038 \\
\hline $\mathrm{Cl}$ & 35 & 1 & 100 & 517.077 & 0.008 & 7.4 & 6.28 \\
\hline $\mathrm{Cl}$ & 35 & 2 & 66.79 & 1164.831 & 0.012 & 8.9 & 4.22 \\
\hline $\mathrm{Cl}$ & 35 & 3 & 48.49 & 788.370 & 0.212 & 5 & 3.12 \\
\hline $\mathrm{Fe}$ & 56 & 1 & 100 & 352.332 & 0.016 & 0.284 & 0.205 \\
\hline $\mathrm{Fe}$ & 56 & 2 & 57.57 & 122.078 & 0.022 & 0.099 & 0.122 \\
\hline $\mathrm{Fe}$ & 56 & 3 & 30.60 & 691.914 & 0.016 & 0.142 & 0.062 \\
\hline $\mathrm{Sb}$ & 121 & 1 & 100 & 121.643 & 0.042 & 0.4 & 0.226 \\
\hline $\mathrm{Sb}$ & 121 & 2 & 76.96 & 61.513 & 0.044 & 0.27 & 0.18 \\
\hline
\end{tabular}

Table 2. List of the elemental composition of SU-8 polymer samples one and two having different solidification and hardening pretreatment

\begin{tabular}{|c|c|c|c|c|c|c|c|c|c|c|}
\hline & \multicolumn{9}{|c|}{ SU-8 sample one } & \multicolumn{4}{c|}{ SU-8 sample two } \\
\hline El. & C \% Atom. & Unc. \% & C \% el/el & Rel.unc. \% & Abs.unc. & C \% Atom. & Unc. \% & C \% el/el & Rel. unc. \% & Abs. unc. \\
\hline \hline $\mathrm{H}$ & 45.6 & 2.3 & 6.0 & 4.4 & 0.3 & 44.7 & 3.1 & 5.7 & 6.0 & 0.3 \\
\hline $\mathrm{C}$ & 42.6 & 2.6 & 67 & 3.8 & 2.6 & 39.9 & 3.5 & 61 & 5.8 & 3.5 \\
\hline $\mathrm{O}$ & 9.9 & 15.9 & 21 & 14.1 & 2.9 & 13.7 & 17.6 & 28 & 14.8 & 4.1 \\
\hline $\mathrm{F}$ & 1.6 & 8.5 & 4.1 & 9.1 & 0.4 & 1.5 & 9.2 & 3.5 & 10.4 & 0.4 \\
\hline $\mathrm{S}$ & 0.091 & 3.5 & 0.38 & 5.0 & 0.02 & 0.090 & 4.1 & 0.37 & 6.6 & 0.02 \\
\hline $\mathrm{Cl}$ & 0.035 & 4.5 & 0.16 & 5.8 & 0.01 & 0.034 & 5.0 & 0.15 & 7.2 & 0.01 \\
\hline $\mathrm{Fe}$ & 0.0095 & 8.3 & 0.07 & 9.0 & 0.01 & 0.0073 & 12.4 & 0.05 & 13.4 & 0.01 \\
\hline $\mathrm{Sb}$ & 0.064 & 3.6 & 1.0 & 5.1 & 0.1 & 0.065 & 4.2 & 1.0 & 6.6 & 0.1 \\
\hline $\mathrm{Pb}$ & 0.029 & 14.9 & 0.8 & 15.2 & 0.1 & 0.029 & 16.9 & 0.8 & 17.6 & 0.1 \\
\hline
\end{tabular}

*SU-8 sample one: soft baked by heating at $90^{\circ} \mathrm{C}$ for 158 hours.

Final solidification using $10 \mathrm{~min}$ UV plus heat at $90^{\circ} \mathrm{C}$ for $40 \mathrm{~min}$

$* S U-8$ sample two: soft bake heated at $90^{\circ} \mathrm{C}$ for 158 hours.

Final solidification using $20 \mathrm{~min} \mathrm{UV}$ and heat at $90^{\circ} \mathrm{C}$ for $20 \mathrm{~min}$.

Rel. unc.: relative uncertainty of the measured value.

Abs. unc.: absolute uncertainty of the measured value. 


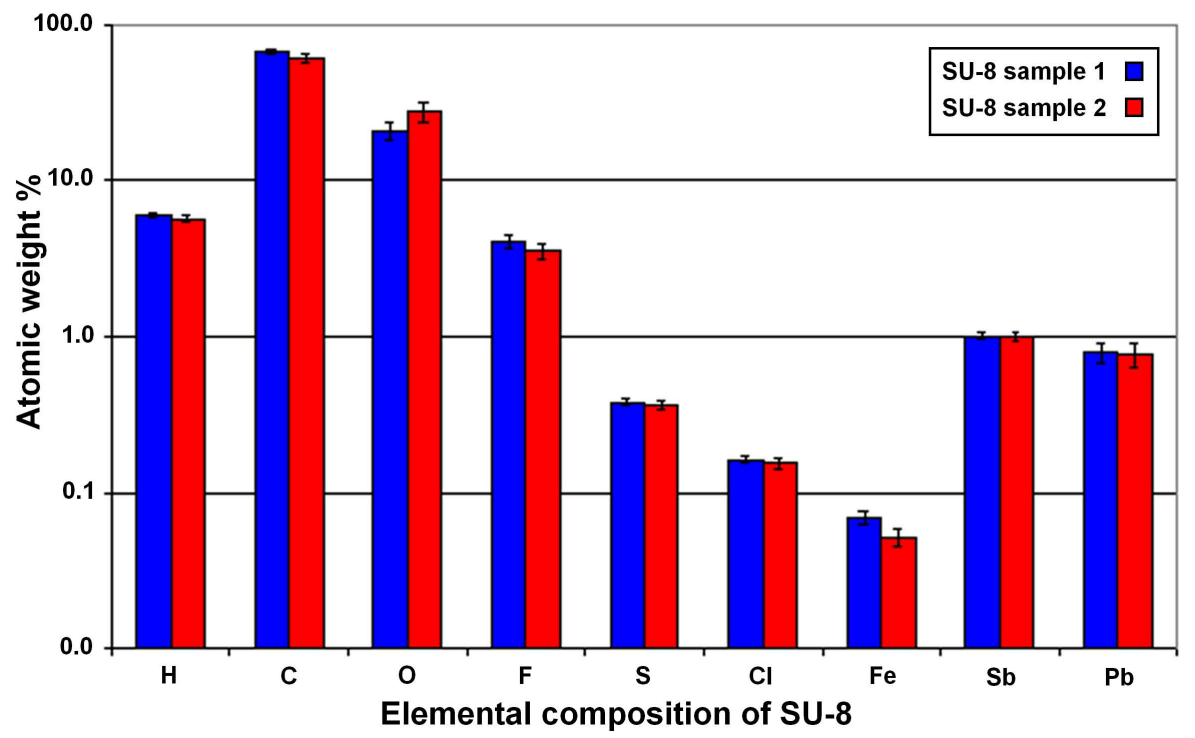

Fig. (4). Graph of atomic weight percentages versus SU-8 elemental composition.

elemental composition. The samples, hence, are of a very consistent and pure composition as essential for producing the stable microturbine preforms shown in Fig. (1a).

\section{CONCLUSIONS}

In this paper, the application of PGAA is tested in the investigation of two SU-8 organic polymer microturbine samples. PGAA has found very useful data in a nondestructive way about the percentages of the major components, and some interesting trace elements within the bulk SU-8. No significant difference between the two samples was found, thus providing a substantial indication for the consistent and pure composition of the material regardless of varying pretreatment and laser heat processing effects. A loss or difference could influence the mechanical properties of the investigated SU-8, especially in the application of making the fine microturbines structures.

The present work is an industrial application of a neutron technique [5], and demonstrates the usefulness of PGAA in the industrial field of polymer investigation. The results confirm that PGAA is a helpful industrial tool that is well suited to the investigation of other polymer materials.

\section{ACKNOWLEDGMENTS}

The authors would like to thank the Tyndall National Institute, Ireland for tests via the National Access Program on the SU-8 samples used in this investigation. Thanks to the EPSRC for their funding for the fabrication and development of the microturbine generator device under grant number
GR/N18895. Finally, sincere thanks to Dr Andrew Holmes at Imperial College London for starting the microturbine generator project and allowing the fabrication of turbines for testing by PGAA.

\section{REFERENCES}

[1] Holmes, A. S.; Hong G.; Heaton, M.E. In Laser precision microfabrication, Proceedings of the 3rd International symposium on LMP 2002, Osaka, Japan, May 27-31, 2002.

[2] Heaton, M. E. Proc. Soc. Photo Opt. Instrum. Eng., 2005, 5824, 216.

[3] Hong, G.; Holmes, A.S.; Heaton, M.E. Microsyst. Techn., 2004, 10, 357.

[4] Lorenz, H.; Despont, M.; Fahmi, N.; La Bianca, N.; Renaud P.; Vettiger, P. J. Micromech. Microeng., 1997, 7, 121.

[5] Rogante, M. In Industrial Applications of Neutron Techniques, Proceedings 1st Italian Workshop for Industry, Civitanova Marche, Italy, June 12-14, 2008; Rogante, M., Ed.; Rogante Engineering, Civitanova Marche, Italy, 2008, pp. 40-120. http://www.roganteengineering.it/aitn2008.html (accessed Dec. 12, 2008).

[6] Rogante, M. In Materials \& Tribology, Procedings of the International Conference MATRIB '03, Vela Luka, Croatia, June 26-28, 2003; Grilec K., Ed.; Croatian Society for Materials and Tribology: Zagreb, Croatia, 2003; pp. 235-241.

[7] Rogante, M.; Rosta, L. Proc. Soc. Photo Opt. Instrum. Eng., 2005, 5824, 294.

[8] Révay, Zs.; Belgya, T.; Kasztovszky, Zs.; Weil, J.L.; Molnár, G.L. Nucl. Instr. Meth. Phys. Res. B, 2004, 213, 385.

[9] Rogante, M. ICP, Reedbusiness, Milano, 2008, $2,72$.

[10] Révay, Zs.; Molnar, G.L.; Belgya, T.; Kasztovszky, Zs.; Firestone, R.B. J. Radioanal. Nucl. Chem., 2001, 248, 395.

Received: November 12, 2008

(C) Heaton et al.; Licensee Bentham Open.

This is an open access article licensed under the terms of the Creative Commons Attribution Non-Commercial License (http://creativecommons.org/licenses/by-nc/3.0/) which permits unrestricted, non-commercial use, distribution and reproduction in any medium, provided the work is properly cited. 